\title{
Certified Adult Nurse Practitioner
}

National Cancer Institute

\section{Source}

National Cancer Institute. Certified Adult Nurse Practitioner. NCI Thesaurus. Code C71286.

A registered nurse who has completed an advanced training program endorsed authoritatively as having met certain requirements in adult medicine. 